\title{
Expression of VE-Cadherin in Peritubular Endothelial Cells during Acute Rejection after Human Renal Transplantation
}

\author{
Ana Roussoulières, ${ }^{1,2}$ Brigitte McGregor, ${ }^{3}$ Lara Chalabreysse, ${ }^{4}$ Catherine Cerutti, ${ }^{1}$ Jeanne-Luce Garnier, ${ }^{5}$ \\ Pascale Boissonnat, ${ }^{2}$ Olivier Bastien, ${ }^{2}$ Jean-Yves Scoazec, ${ }^{3}$ Françoise Thivolet-Bejui, ${ }^{4}$ Laurent Sebbag, ${ }^{2}$ \\ and John L. McGregor ${ }^{1,6}$ \\ ${ }^{1}$ INSERM U331/EA 3740, Faculté de Médecine RTH Laennec, 8 rue Guillaume Paradin, 69003 Lyon, France \\ ${ }^{2}$ Department of Cardiac Transplantation, Hôpital Cardiologique Louis Pradel, 28 avenue du Doyen Lépine, 69003 Lyon, France \\ ${ }^{3}$ Department of Pathology, Hôpital Edouard Herriot, 5 place d'Arsonval, 69003 Lyon, France \\ ${ }^{4}$ Department of Pathology, Hôpital Cardiologique Louis Pradel, 28 avenue du 4 Doyen Lépine, 69003 Lyon, France \\ ${ }^{5}$ Department of Nephrology, Hôpital Edouard Herriot, 5 place d'Arsonval, 69003 Lyon, France \\ ${ }^{6}$ Centre for Cardiovascular Biology \& Medicine, King's College London, Strand WC2R 2LS, UK
}

Correspondence should be addressed to Ana Roussoulières, ana.roussoulieres@chu-lyon.fr

Received 29 November 2006; Accepted 10 May 2007

Recommended by Abdelali Haoudi

Genes involved in acute rejection (AR) after organ transplantation remain to be further elucidated. In a previous work we have demonstrated the under-expression of VE-Cadherin by endothelial cells (EC) in AR following murine and human heart transplantation. Serial sections from 15 human kidney Banff-graded transplant biopsies were examined for the presence of VE-Cadherin and CD34 staining by immunohistochemistry (no AR $(n=5)$, AR grade IA $(n=5)$, or AR grade IIA $(n=5))$. Quantification of peritubular EC staining were evaluated and results were expressed by the percentage of stained cells per surface analysed. There was no difference in CD34 staining between the 3 groups. VE-Cadherin expression was significantly reduced in AR Grade IIA when compared to no $\operatorname{AR}(P=.01)$ and to AR grade IA $(P=.02)$. This study demonstrates a reduced VE-Cadherin expression by EC in AR after renal transplantation. The down-regulation of VE-Cadherin may strongly participate in human AR.

Copyright ( 92007 Ana Roussoulières et al. This is an open access article distributed under the Creative Commons Attribution License, which permits unrestricted use, distribution, and reproduction in any medium, provided the original work is properly cited.

\section{INTRODUCTION}

Acute rejection, observed after renal transplantation, has a significant impact for long-term renal allograft survival. Even though its incidence was reduced by new immunosuppressive drugs, it remains a major problem after renal transplantation. Acute rejection is a cell-mediated immune response that is initiated by the recognition of $\mathrm{CD}^{4+} \mathrm{T}$ cells by the major histocompatibility complex (MHC) class II antigens on antigen-presenting cells (APCs) of the graft [1-4]. MHC class II antigen activates $\mathrm{CD}^{4+} \mathrm{T}$-lymphocytes, which subsequently release cytokines. Released cytokines target vascular endothelial cells, the first cells to be recognized by the host's immune system, and induce the expression of adhesion molecules and chemokines implicated in T-lymphocyte adhesion and extravasation. Initial transient adhesion of Tlymphocytes mediated principally by selectins induce the rolling of the T-cells and their subsequent activation and adhesion on the endothelium via other adhesion molecules such as ICAM-1 and VCAM- 1 . This adhesion stage provides the necessary signal for full T-cell activation leading to Tlymphocytes transendothelial migration [5].

Endothelial cells forming the interface between donor and recipients are the first donor cells to be recognized by the host's immune system. Endothelial cells coating the capillaries that act as a barrier between the donor organ and recipient bears the MHC class II molecules. They are highly responsive to cytokines and express adhesion and other molecules implicated in T-lymphocyte adhesion and extravasation. Such endothelial control in cell migration requires an effective intercellular adhesion, so called cell junction, between the endothelial cells. Morphologically, three types of organelles constitute the endothelial cell junctions [6]. Tight junctions seal the cells to each other. Gap junctions 
TABle 1: Patients demographics.

\begin{tabular}{|c|c|c|c|c|}
\hline Characteristics & $\begin{array}{l}\text { No acute rejection } \\
\qquad(n=5)\end{array}$ & $\begin{array}{l}\text { Acute rejection grade IA } \\
\qquad(n=5)\end{array}$ & $\begin{array}{l}\text { Acute rejection grade IIA } \\
\qquad(n=5)\end{array}$ & $P$ value \\
\hline Recipient age (years) & $55 \pm 13$ & $55 \pm 4$ & $32 \pm 12$ & .02 \\
\hline Recipient sex (M/F) & $1 / 4$ & $5 / 0$ & $4 / 1$ & .1 \\
\hline Donor age (years) & $54 \pm 20$ & $34 \pm 20$ & $32.6 \pm 14$ & .1 \\
\hline Donor sex (M/F) & $4 / 1$ & $3 / 2$ & $4 / 1$ & .9 \\
\hline Ischemic time $(\mathrm{h})$ & $17.9 \pm 3.6$ & $15.4 \pm 3.7$ & $17.1 \pm 1.0$ & .2 \\
\hline Time between & & & & \\
\hline $\begin{array}{l}\text { Transplantation and } \\
\text { biopsy (days) }\end{array}$ & $2.8 \pm 1.9$ & $1244.8 \pm 2037.7$ & $270.9 \pm 178.3$ & .01 \\
\hline Serum creatinine $(\mu \mathrm{mol} / \mathrm{L})$ & $238.3 \pm 137.1$ & $212 \pm 77.45$ & $286.4 \pm 150.11$ & .7 \\
\hline
\end{tabular}

allow exchange of ions and small molecules between adjacent cells. Adherent junctions mediate the physical contacts between cells and anchor the actin cytoskeleton. Cell-cell adhesion structures have been studied and some membrane proteins have been described such as PECAM-1 or CD31 [7]; $\alpha 5 \beta 1$ and $\alpha 2 \beta 1$ integrins [8], V-Cadherin [9], and vascular endothelium cadherin (VE-Cadherin) [10-12]. The extravasation of lymphocytes in rejecting renal allografts is thought to take place in activated peritubular capillaries [13].

To date, the exact mechanisms involved in acute rejection after solid organ transplantation are not completely understood. In a previous work [14] we have demonstrated that VE-Cadherin is under expressed in acute rejection in a murine heterotopic heart transplantation model and in acute rejection following human heart transplantation. Murine macroarrays results were validated in mice and in humans by immunohistochemistry and quantitative real-timepolymerase chain reaction (Q-PCR). In this study we have performed immunohistochemical staining of VE-Cadherin in human biopsies after renal transplantation. We demonstrate that VE-Cadherin is also under expressed in acute rejection after renal transplantation.

\section{MATERIALS AND METHODS}

\subsection{Patients}

This study comprised transplant biopsy specimens from 15 patients ( $66 \%$ of men with mean age of $47 \pm 15$ years). All patients received deceased donor kidney transplants. After transplantation, all patients received induction therapy with anti-lymphocyte globulin or anti IL-2 receptor and triple-therapy immunossupression (cyclosporine, steroids, and mycophenolate mofetil). Details relating to important clinical parameters in each group are given in Table 1.

\subsection{Renal Specimens}

Needle kidney transplant biopsies were performed solely to define the diagnosis and management of patients with acute deterioration of allograft function in accordance with institutional guidelines. Renal allograft tissues were obtained from transplant recipients undergoing ultrasound guided biopsies from December 2002 to August 2003. Biopsies were performed between 1 day and 1-6 years after renal transplantation (453, $4 \pm 1118,8$ days). Specimens were formalin fixed and paraffin embedded or snap frozen in liquid nitrogen. Fifteen renal transplant biopsy specimens were examined.

\subsection{Histopathological study}

Paraffin sections $(4 \mu \mathrm{m})$ were stained with Periodic acid Schiff (PAS) and graded for acute rejection using the Banff criteria [15]. Fifteen kidney transplant biopsies showing no rejection $(n=5)$, acute rejection grade IA $(n=5)$, or acute rejection grade IIA $(n=5)$ were examined.

\subsection{Immunohistochemistry}

Serial sections were used for VE-Cadherin and CD34 immunostaining. Negative controls were performed with the use of irrelevant isotype matched antibodies. Positive control was performed using human spleen tissues that show a large number of endothelial cells.

\subsubsection{VE-Cadherin}

Sections were incubated for 1 hour with a mouse antihuman VE-Cadherin monoclonal antibody (1:50, Chemicon International, Temecula, CA, USA) followed by a goat antimouse immunoglobulin (Dako, France), which was then revealed by the streptavidin-biotin immunoperoxidase method.

\subsection{2. $C D 34$}

Sections were incubated for 1 hour with a mouse anti-human CD34 monoclonal antibody (1:50, Dako, France) followed by an antimouse biotin (Dako, France) which was then revealed by the streptavidin-biotin immunoperoxidase method.

\subsection{Quantitative analysis}

Sections were assessed by two independent observers (AR, $\mathrm{BM})$, scored according to the number of VE-Cadherin stained endothelial cells in peritubular capillaries and the number of CD34 stained cells. Sections were analyzed with a Leica DMLB microscope (Leica Microsystems, Germany) 


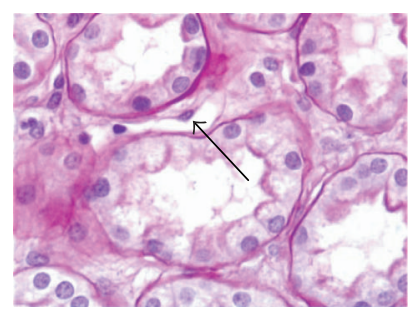

(a)

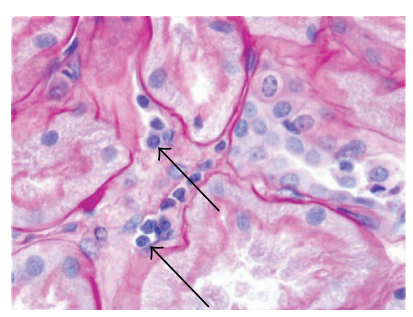

(b)

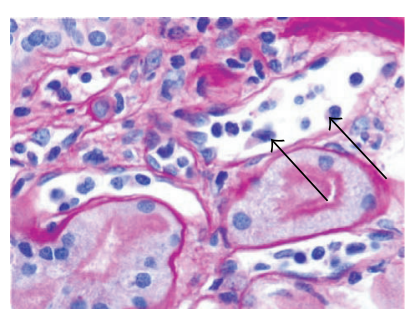

(c)

FIGURE 1: Periodic acid schiff (PAS) staining (X 400) of renal biopsies. (a) No acute rejection, (b) acute rejection grade IA, (c) acute rejection grade IIA. Arrows show peritubular capillary endothelial cells.

directly coupled to a 3CCD colour camera (JVC, Argenteuil, France). Quantification of VE-Cadherin and CD34 staining was evaluated using image analysis software (Perfect Image, Claravision Orsay, France). Positive VE-Cadherin and CD34 labelled peritubular endothelial cells were counted in 10 high-power fields for each sample (X630 magnification). Results were expressed by the percentage of surface labelled stained cells. Mean values were obtained for each specimen and used for statistical analysis.

\subsection{Statistical analysis}

Kruskal-Wallis nonparametric test was used to compare clinical characteristics between the 3 groups. In order to take into account the variability between fields for each sample, variance analysis for repeated measures was used to compare VE-Cadherin immunohistochemical staining between the 3 groups. When this global test was significant, Fisher LSD post-hoc test was further used when comparing between 2 groups. $P$ values $<.05$ were considered to indicate statistically significant differences.

\section{RESULTS}

\subsection{Patients characteristics}

The demographics are summarized in Table 1. The mean time between transplantation and allograft biopsy was $431 \pm$ 1081 days (range 1-4298 days). There was no statistical difference between donor age, donor and recipient sex or ischemic time. Serum creatinine (mean \pm SD) was not statistically different between groups. All patients with histopathologic diagnosis of acute rejection received steroid treatment after the biopsy was obtained.

\subsection{Histopathological evaluation}

All human biopsies were constituted by adequate fragments according to Banff criteria [15] showing no acute rejection $(n=5)$, grade IA acute rejection $(n=5)$, or grade IIA acute rejection $(n=5)$ (see Figure 1$)$. In the no acute rejection group the peritubular capillary endothelial cells have a normal shape and an elongated cytoplasm. In the acute rejection grade IA the lumen size is unchanged but populated with numerous inflammatory cells adhering to swollen endothelial cells. In the acute rejection IIA, the lumen is di- lated, some swollen endothelial cells seemed to be detached from the basement membrane and inflammatory cells have migrated from the lumen to the interstitium.

\subsection{Immunohistochemistry}

There was no difference in CD34 staining between the 3 groups. VE-Cadherin staining was differentially expressed between the 3 groups $(P=.02)$. In no acute rejection group VE-Cadherin stained cells represented $1.65 \pm 0.71 \%$ of the surface examined compared to $1.62 \pm 0.34 \%$ in acute rejection grade IA and $0.75 \pm 0.31 \%$ in acute rejection grade IIA (see Figure 2). VE-Cadherin expression was significantly reduced in acute rejection grade IIA when compared to no acute rejection $(P=.01)$ and to acute rejection grade IA $(P=.02)$. There was no difference in VE-Cadherin expression between acute rejection grade IA and no acute rejection group $(P=.7)$. Figure 3 shows VE-Cadherin staining as a strong, thin, linear staining on peritubular endothelial cells in renal biopsies showing no rejection or acute rejection grade IA compared to acute rejection grade IIA. CD34 staining confirmed the staining of VE-Cadherin on peritubular endothelial cells (see Figure 3).

\section{DISCUSSION}

The working hypothesis in this study is that the endothelium lining the vessel wall of the donor kidney is first to be in contact with host cells and is subjected to immunological interactions. Such interactions result in the over and under expression of some molecules. These molecules can facilitate the adhesion and transmigration of lymphocytes in the renal tissue leading to damage and ultimately to graft failure. We have previously demonstrated by macroarrays, immunohistochemistry, and Q-PCR that VE-Cadherin is under expressed in acute rejection following murine and human heart transplantation [14]. In the current investigation, we bring evidence for the first time that VE-Cadherin is implicated also in acute rejection following renal transplantation.

Lymphocyte adhesion to the vascular lining accompanies the first stages of the acute rejection reaction. The ability of endothelial cells to adhere lymphocytes is not a constant or a static phenomenon. Several cytokines and adhesion molecules have shown to increase lymphocyte binding to and penetration through endothelial cells [16]. In response 


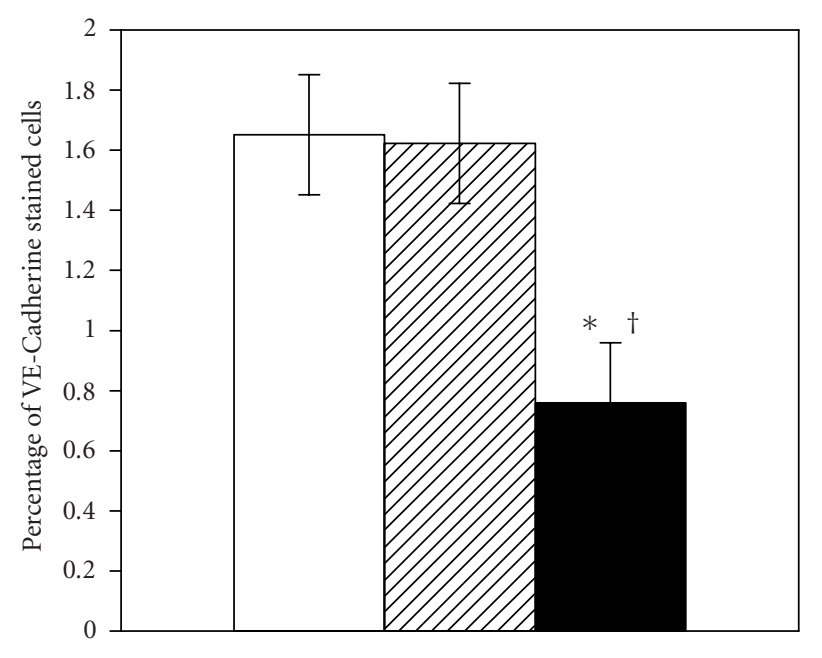

$\square$ No acute rejection
$\square$ Acute rejection grade IA
Acute rejection grade IIA

FIGURE 2: Immunohistochemical staining grade of VE-Cadherin on endothelial cells. ${ }^{*} P<.05$ acute rejection grade IIA versus acute rejection grade $\mathrm{IA} ;{ }^{\dagger} P<.05$ acute rejection grade IIA versus no acute rejection.

to cytokines and to the expression of endothelial cells adhesion molecules, leukocytes first roll on the endothelial surface. Binding to endothelium is necessary, but not a sufficient request for the leukocyte to get into tissue. This stage is followed by a firm adhesion, and then by a rapid transmigration of leukocytes through endothelial intercellular junctions. Renkonen et al. [13] suggested and confirmed the hypothesis that the peritubular capillary endothelium is the site of entry of lymphocytes into rejecting kidney allografts. The authors showed that the increase in lymphocyte binding to peritubular capillaries precede the peak of leukocyte accumulation in the graft. Light and electron microscopy revealed a marked activation of peritubular capillary endothelial cells in allografts, whereas these alterations were less severe or absent in syngenic controls and normal kidneys.

VE-Cadherin is an endothelial-specific membrane protein, present in adherent junctions of endothelial cells and responsible for the endothelial cell-cell adhesion. Suzuki et al. [10] first described it and it was initially denoted cadherin5. Lampugnani et al. [11] studied cultured endothelial cells monolayers by immunofluorescence microscopy. They described VE-Cadherin as an endothelial-specific membrane protein with a thin, sharp continuous line highlighting the margins of each cell. It was present at the appositional surfaces of cultured cells only on reaching confluency. They observed that if the endothelial permeability was increased its distribution was punctuated and could be found only at intercellular contacts in a few areas. Since then, various authors demonstrated the properties of cell-cell adhesion of the VECadherin and the relationship of its diminution with the increase of the endothelial permeability [17-20].

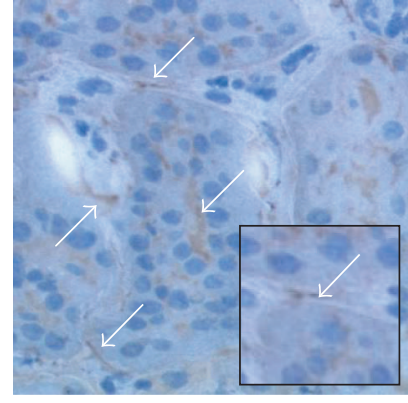

VE-Cadherin

(a)

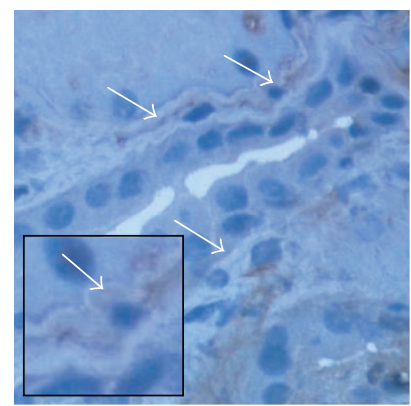

VE-Cadherin

(c)

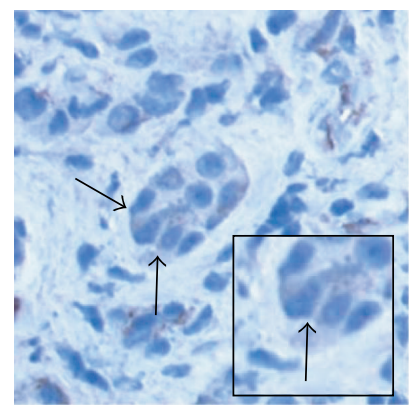

VE-Cadherin

(e)

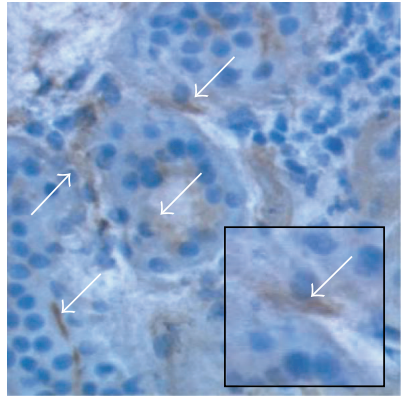

CD 34

(b)

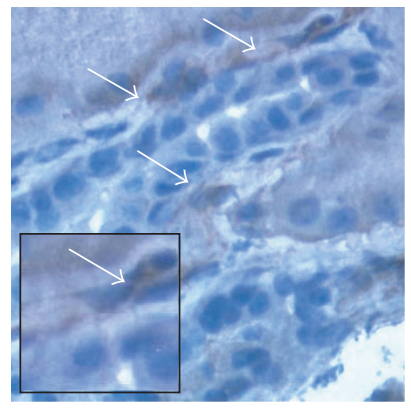

CD 34

(d)

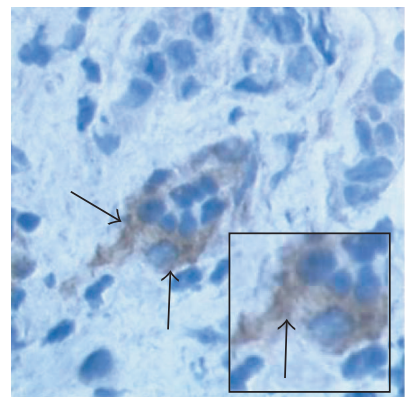

CD 34

(f)
Figure 3: VE-Cadherin and CD34 immunohistochemical staining of frozen renal biopsy serial sections (X 400). (a), (b) No acute rejection. (c), (d) Acute rejection grade IA. (e), (f) Acute rejection grade IIA. (a), (c) and (e): VE-Cadherin staining on peritubular endothelial cells (arrows). (b), (d) and (f): CD34 staining on peritubular endothelial cells (arrows).

In our study, we found the same kind of staining described as a thin, continuous line in the margins of renal peritubular human endothelial cells. Cell adhesion molecules, mediating leukocyte adhesion to endothelial cells, have been shown to affect gene transcription in these cells. Previous studies $[1,11,12]$ have demonstrated that through their adhesion, leukocyte could transfer intracellular signals to endothelial cells in different ways. Del Maschio et al. [21] suggested that these intracellular signals could induce endothelial intercellular disorganisation. They found that leukocyte adhesion to endothelial cells was related to 
a reduced VE-Cadherin expression that could increase endothelial permeability. An alternative explanation for adherent junction's disorganisation is the release of lytic enzymes from the PMN that can be responsible for VE-Cadherin digestion and junction disassembly. VE-Cadherin is particularly susceptible to proteolytic digestion [11]. Such potential release of proteolytic enzymes [22], by activated leukocytes, may lead to cleavage of the extracellular domain of VECadherin, and an increase in the permeability in areas bearing deposited leukocytes. Moreover, other authors [23, 24] have reported changes in cytosolic $\mathrm{Ca}^{2+}$ level in endothelial cells during the adhesion of polymorphonuclear leukocyte. The polymorphonuclear leukocyte adherence could induce a series of endothelial intracellular responses leading a detachment of catenins from VE-Cadherin. It is conceivable that in acute rejection after solid organ transplantation activated lymphocytes adhering to vascular endothelial cells could affect the latter cells by inducing the disappearance of VECadherin from endothelial adherent junctions. Such action could result in a significant increase in endothelial permeability due to the disassembly of endothelial adherent junctions.

VE-Cadherin was also investigated in other settings than acute rejection after solid organ transplantation. Sutton et al. [25] studied the immunohistochemical expression of VECadherin in an animal model of renal ischemia. Renal ischemia was induced by clamping the renal pedicule for 32 minutes. The authors observed that 24 hours after ischemia, the majority of the renal microvasculature did not stain for VE-Cadherin. They observed also that 72 hours after ischemia, VE-Cadherin staining in the renal microvasculature was similar to that observed under physiological conditions. Cerini et al. [26] studied the in vitro effect of the uremic retention solute $\mathrm{p}$-cresol observed in chronic renal failure. They observed an increase in endothelial permeability associated with a decreased staining of junctional VE-Cadherin.

In summary, in this study we have demonstrated for the first time a correlation between the expression of VECadherin present in peritubular endothelial cells and acute allograft rejection after human renal transplantation. The under expression of VE-Cadherin in peritubular capillaries in acute rejection after kidney transplantation could be responsible for the lymphocyte transmigration into interstitial tissues leading to graft dysfunction. Preventing the down regulation of VE-Cadherin could conceivably prevent severe acute graft rejection.

\section{ACKNOWLEDGMENTS}

The authors would like to thank Association pour le Développement de la Greffe Cardiaque and Mary and Garry Weston Foundation.

\section{REFERENCES}

[1] M. L. Rose, "Endothelial cells as antigen-presenting cells: role in human transplant rejection," Cellular and Molecular Life Sciences, vol. 54, no. 9, pp. 965-978, 1998.
[2] A. S. Daar, S. V. Fuggle, J. W. Fabre, A. Ting, and P. J. Morris, "The detailed distribution of MHC class II antigens in normal human organs," Transplantation, vol. 38, no. 3, pp. 293-298, 1984.

[3] M. L. Rose, M. I. Coles, R. J. Griffin, A. Pomerance, and M. H. Yacoub, "Expression of class I and class II major histocompatibility antigens in normal and transplanted human heart," Transplantation, vol. 41, no. 6, pp. 776-780, 1986.

[4] C. Page, M. Rose, M. Yacoub, and R. Pigott, "Antigenic heterogeneity of vascular endothelium," American Journal of Pathology, vol. 141, no. 3, pp. 673-683, 1992.

[5] T. A. Springer, "Traffic signals for lymphocyte recirculation and leukocyte emigration: the multistep paradigm," Cell, vol. 76, no. 2, pp. 301-314, 1994.

[6] E. Dejana, M. Corada, and M. G. Lampugnani, "Endothelial cell-to-cell junctions," The FASEB Journal, vol. 9, no. 10, pp. 910-918, 1995.

[7] D. L. Simmons, C. Walker, C. Power, and R. Pigott, "Molecular cloning of CD31, a putative intercellular adhesion molecule closely related to carcinoembryonic antigen," Journal of Experimental Medicine, vol. 171, no. 6, pp. 2147-2152, 1990.

[8] M. G. Lampugnani, M. Resnati, E. Dejana, and P. C. Marchisio, "The role of integrins in the maintenance of endothelial monolayer integrity," Journal of Cell Biology, vol. 112, no. 3, pp. 479-490, 1991.

[9] R. L. Heimark, M. Degner, and S. M. Schwartz, "Identification of a $\mathrm{Ca}^{2+}$-dependent cell-cell adhesion molecule in endothelial cells," Journal of Cell Biology, vol. 110, no. 5, pp. 1745-1756, 1990.

[10] S. Suzuki, K. Sano, and H. Tanihara, "Diversity of the cadherin family: evidence for eight new cadherins in nervous tissue," Cell regulation, vol. 2, no. 4, pp. 261-270, 1991.

[11] M. G. Lampugnani, M. Resnati, M. Raiteri, et al., "A novel endothelial-specific membrane protein is a marker of cell-cell contacts," Journal of Cell Biology, vol. 118, no. 6, pp. 15111522, 1992.

[12] M. G. Lampugnani, M. Corada, L. Caveda, et al., "The molecular organization of endothelial cell to cell junctions: differential association of plakoglobin, $\beta$-catenin, and $\alpha$-catenin with vascular endothelial cadherin (VE-cadherin)," Journal of Cell Biology, vol. 129, no. 1, pp. 203-217, 1995.

[13] R. Renkonen, J. P. Turunen, J. Rapola, and P. Hayry, "Characterization of high endothelial-like properties of peritubular capillary endothelium during acute renal allograft rejection," American Journal of Pathology, vol. 137, no. 3, pp. 643-651, 1990.

[14] A. Roussoulières, O. Raisky, L. Chalabreysse, et al., "Identification and characterization of two genes (MIP- $1 \beta$, VEcadherin) implicated in acute rejection in human heart transplantation-use of murine models in tandem with cDNA arrays," Circulation, vol. 111, no. 20, pp. 2636-2644, 2005.

[15] L. C. Racusen, K. Solez, R. B. Colvin, et al., "The Banff 97 working classification of renal allograft pathology," Kidney International, vol. 55, no. 2, pp. 713-723, 1999.

[16] T. A. Springer, "Traffic signals on endothelium for lymphocyte recirculation and leukocyte emigration," Annual Review of Physiology, vol. 57, pp. 827-872, 1995.

[17] S. D. Trocha, C. G. Kevil, M. C. Mancini, and J. S. Alexander, "Organ preservation solutions increase endothelial permeability and promote loss of junctional proteins," Annals of Surgery, vol. 230, no. 1, pp. 105-113, 1999. 
[18] M. Corada, F. Liao, M. Lindgren, et al., "Monoclonal antibodies directed to different regions of vascular endothelial cadherin extracellular domain affect adhesion and clustering of the protein and modulate endothelial permeability," Blood, vol. 97, no. 6, pp. 1679-1684, 2001.

[19] C. Bianchi, E. G. Araujo, K. Sato, and F. W. Sellke, "Biochemical and structural evidence for pig myocardium adherens junction disruption by cardiopulmonary bypass," Circulation, vol. 104, supplement 1, pp. I319-I324, 2001.

[20] S. K. Shaw, P. S. Bamba, B. N. Perkins, and F. W. Luscinskas, "Real-time imaging of vascular endothelial-cadherin during leukocyte transmigration across endothelium," Journal of Immunology, vol. 167, no. 4, pp. 2323-2330, 2001.

[21] A. Del Maschio, A. Zanetti, M. Corada, et al., "Polymorphonuclear leukocyte adhesion triggers the disorganization of endothelial cell-to-cell adherens junctions," Journal of Cell Biology, vol. 135, no. 2, pp. 497-510, 1996.

[22] S. J. Weiss, “Tissue destruction by neutrophils," New England Journal of Medicine, vol. 320, no. 6, pp. 365-376, 1989.

[23] E. C. Butcher, "Leukocyte-endothelial cell recognition: three (or more) steps to specificity and diversity," Cell, vol. 67, no. 6, pp. 1033-1036, 1991.

[24] D. H. Adams and S. Shaw, "Leucocyte-endothelial interactions and regulation of leucocyte migration," Lancet, vol. 343, no. 8901, pp. 831-836, 1994.

[25] T. A. Sutton, H. E. Mang, S. B. Campos, R. M. Sandoval, M. C. Yoder, and B. A. Molitoris, "Injury of the renal microvascular endothelium alters barrier function after ischemia," American Journal of Physiology, vol. 285, no. 2, pp. F191-F198, 2003.

[26] C. Cerini, L. Dou, F. Anfosso, et al., "P-cresol, a uremic retention solute, alters the endothelial barrier function in vitro," Thrombosis and Haemostasis, vol. 92, no. 1, pp. 140-150, 2004. 


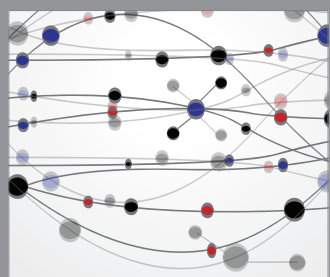

The Scientific World Journal
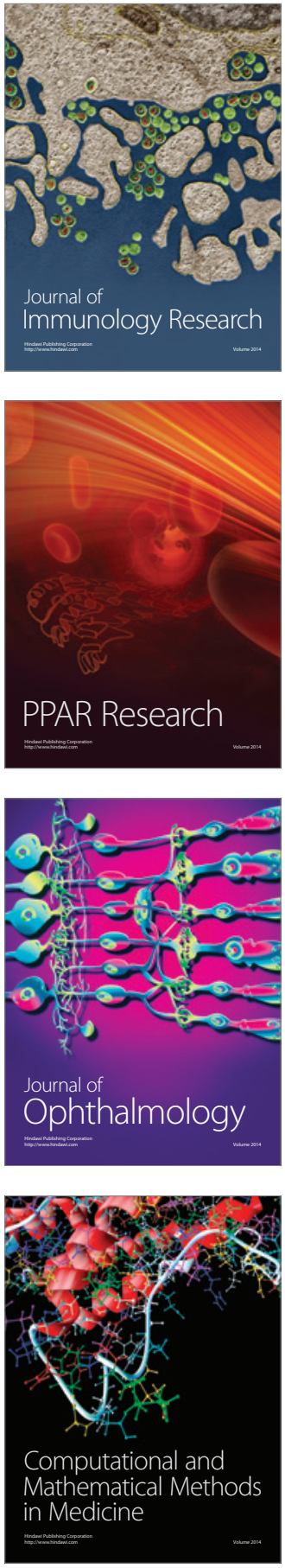

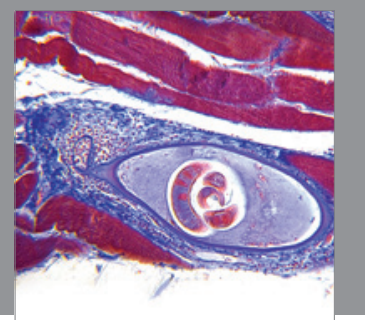

Gastroenterology

Research and Practice
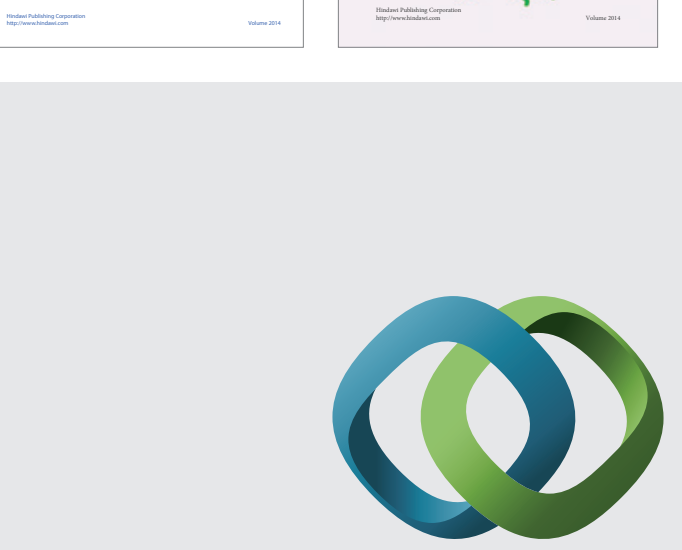

\section{Hindawi}

Submit your manuscripts at

http://www.hindawi.com
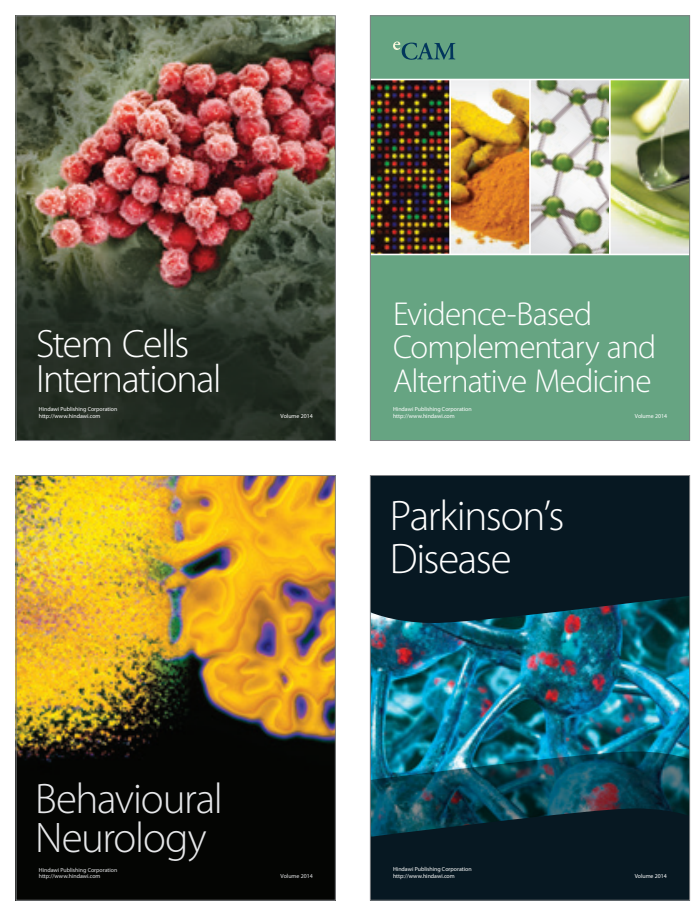

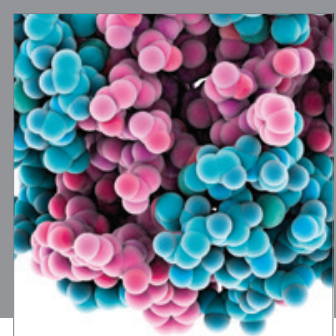

Journal of
Diabetes Research

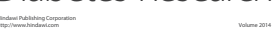

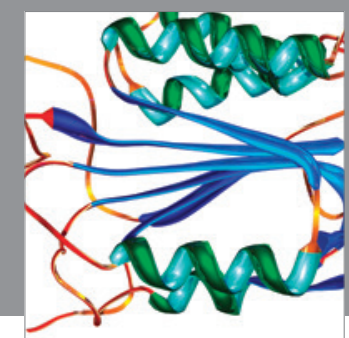

Disease Markers
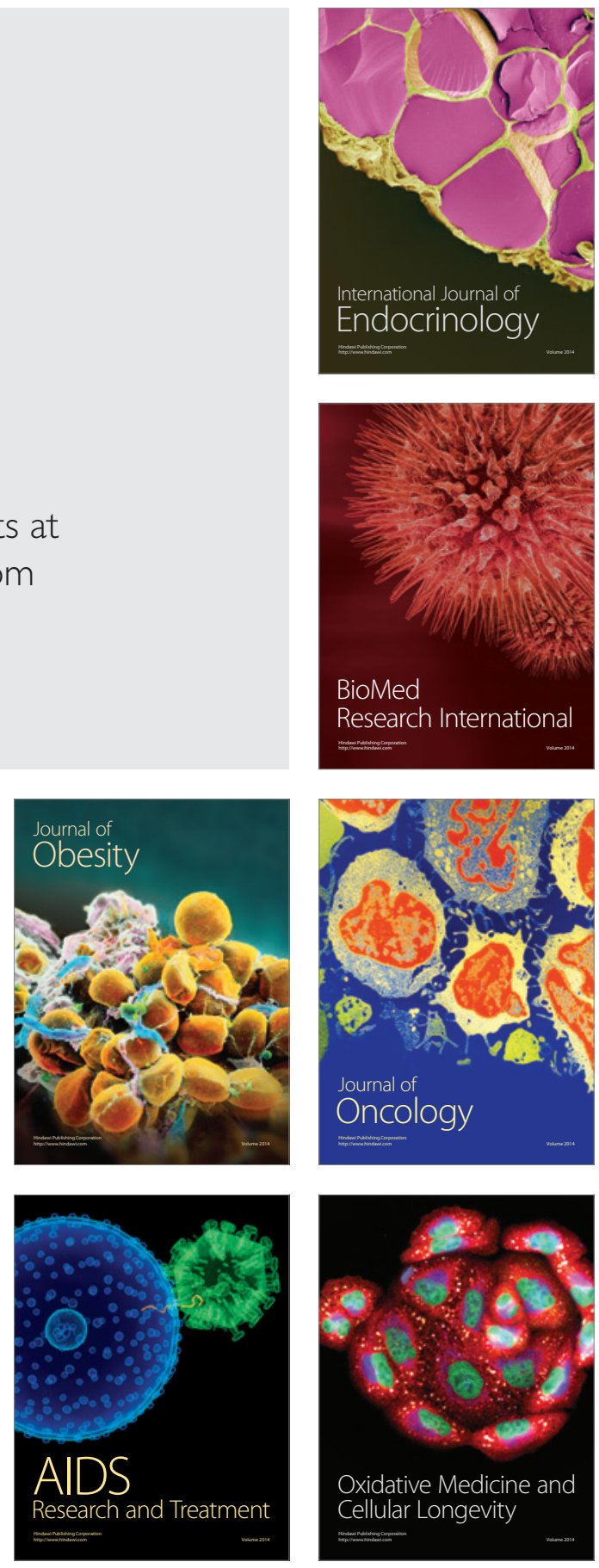